\title{
ESPECIES DE ESPONJAS MARINAS CONOCIDAS PARA LA ISLA HISPANIOLA
}

\author{
Alejandro Herrera-Moreno ${ }^{1}$, Liliana Betancourt ${ }^{1}$ y Pedro M. Alcolado ${ }^{2}$ \\ ${ }^{1}$ Programa EcoMar, Inc. Sarasota 121, Bella Vista, Santo Domingo, República Dominicana \\ ${ }^{2}$ Instituto de Oceanología. Ave. 1ra. 18406, Reparto Flores, Playa, La Habana, Cuba
}

\section{RESUMEN}

Se ofrece una cronología histórica de las investigaciones sobre las esponjas de la Isla Hispaniola desde 1864 al presente. La información proviene de los resultados de expediciones y proyectos nacionales e internacionales en más de 20 localidades dominicanas y haitianas, colecciones de cinco museos que albergan 41 especies, y más de 40 publicaciones taxonómicas y ecológicas. De este análisis, se conocen ahora 138 especies de esponjas para Hispaniola: 127 para República Dominicana (lo cual añade unas 102 especies al último inventario nacional), 63 especies para Haití y una especie sin especificar el país. Seis especies, Axinella corrugata, Pseudotrachya amaza, Polymastia tenax, Xestospongia dominicana, Prosuberites psammophilus y Ecionemia dominicana, tienen como tipo una localidad dominicana. Las especies compiladas son representativas de los ecosistemas de manglares, sustratos particulados con macrovegetación y/o pastos marinos y arrecifes coralinos desde la orilla hasta aproximadamente $40 \mathrm{~m}$ de profundidad.

Palabras clave: biodiversidad marina, esponjas marinas, Hispaniola.

Tittle: Marine sponges from Hispaniola Island.

\section{ABSTRACT}

A historical chronology of research reports on Hispaniola sponges, since 1864 until now is presented. Information comes from results of national and international expeditions and projects in more than 20 Dominican and Haitian localities, collections of five museums that harbor 41 species, and more than 40 taxonomic and ecological publications. From this analysis 138 sponge species are now known to be reported for Hispaniola: 127 for Dominican Republic (which adds 102 species to the last national inventory), 63 species for Haiti and one species of unspecified country. Six species, Axinella corrugata, Pseudotrachya amaza, Polymastia tenax, Xestospongia dominicana, Prosuberites psammophilus and Ecionemia dominicana, have Dominican type localities. The compiled species are representative of mangroves, seagrass beds and coral reefs from the shore down to about $40 \mathrm{~m}$ deep.

Key words: marine biodiversity, marine sponges, Hispaniola.

\section{INTRODUCCIÓN}

Más de un siglo de aportes al conocimiento de los poríferos de la Isla Hispaniola se encuentran diseminados en las colecciones de varios museos, reportes de proyectos, tesis de grado y diversas publicaciones. La necesidad de una revisión de esta información dispersa en tiempo y espacio, y su debida actualización a la luz de los últimos cambios taxonómicos, es un reto si queremos evaluar sobre bases científicas el nivel de conocimiento de la biota marina de la Isla para el uso y conservación de una biodiversidad cuya riqueza de especies es prácticamente 
desconocida.

En las Antillas Mayores, existe un catálogo de esponjas para Cuba (Alcolado, 2002) pero hasta donde conocemos no se ha realizado nunca un trabajo similar para Hispaniola, si bien existe una recopilación preliminar de 36 especies para la República Dominicana (CIBIMA, 1992). Con la implementación del Proyecto HISPABIOTA MARINA del Programa EcoMar, Inc. en República Dominicana (Herrera-Moreno y Betancourt, 2011) se realizó una primera compilación y revisión bibliográfica y taxonómica de especies, incluidas las esponjas, con un enfoque histórico e insular (Herrera-Moreno y Betancourt, 2005). Ese primer inventario se amplía en esta revisión, que presenta una lista actualizada de los poríferos de la Isla Hispaniola.

\section{OBJETIVO}

- Ofrecer la primera lista referenciada de los poríferos de la Isla Hispaniola, con indicaciones de los registros para cada uno de los dos países que la integran: Haití y República Dominicana.

\section{MATERIALES Y MÉTODOS}

Para la elaboración del presente trabajo se realizó una búsqueda de publicaciones orientadas hacia la taxonomía y ecología de las esponjas marinas o inventarios originales derivados de proyectos enfocados al estudio de la biodiversidad marina. Asimismo, se realizó una revisión de la información de varios museos, hallándose reportes de interés para este trabajo en las colecciones en línea del Museo Nacional de Historia Natural del Instituto Smithsoniano (NMNH, 2011), el Museo de Zoología Comparativa de Michigan (MCZ, 2011) y el Museo Zoológico de Ámsterdam (ZMA, 2011). Pulitzer-Finali (1986) ofrece información acerca de los poríferos de Hispaniola en el Museo de Génova MSNG y Reiswig (2002) en el de Gran Bretaña BMNH.

Los registros analizados fueron divididos en aquellos correspondientes a localidades dominicanas y haitianas, pero solo para propósitos de orientación del nivel de conocimiento en cada país, pues el enfoque de nuestro trabajo es totalmente insular. El arreglo general de órdenes y familias en la lista de especies, así como la actualización de los nombres científicos sigue básicamente los criterios del World Porifera Database (Soest et al., 2011) complementados por Pedro M. Alcolado. En cada especie se indican entre corchetes las referencias en las que fueron mencionadas, identificando con una $\mathrm{D}$, una $\mathrm{H}$ o $\mathrm{HI}$ si el reporte pertenece, respectivamente, a República Dominicana, Haití o Hispaniola, sin distinción de país. Junto a la referencia se indica, si procede, la abreviatura del museo y al menos un número de colección. Para las especies en que se realizó alguna corrección o enmienda, se aclara con una nota al pie, el nombre con que aparece en el reporte original hallado en la literatura consultada, a fin de que nuestras actualizaciones taxonómicas puedan ser confrontadas.

\section{RESULTADOS Y DISCUSIÓN}

Las investigaciones sobre los poríferos de la Isla Hispaniola tienen entre sus antecedentes más antiguos la obra sobre las esponjas del Mar Caribe de Duchassaing y Michelotti (1864), con dos especies reportadas para la Isla de Santo Domingo (Ciocalypta alleni y Amphimedon compressa), sin que quede claro en qué país fueron colectadas. Dichas especies fueron revisadas posteriormente por De Laubenfels (1936) y recogidas por Soest et al. (1983) en el Catálogo de la colección de esponjas de Duchassaing y Michelotti de las Indias Occidentales. Hyatt (1877) en su revisión de los poríferos de Norteamérica menciona dos especies para Haití. Carter (1879), en su descripción de Asteropus simplex, incluye material haitiano del Museo de Liverpool. 
En febrero de 1933, durante la Expedición Johnson-Smithsonian Deep Sea en el Yate Caroline, se realizaron colectas en dos estaciones de la Bahía de Samaná (Estaciones 51 y 52), República Dominicana, en profundidades de 10 a $37 \mathrm{~m}$. Se reportaron siete especies de esponjas, dos de las cuales tienen como localidad tipo a la Bahía de Samaná: Axinella corrugata (George y Wilson, 1919) y Pseudotrachya amaza (de Laubenfels, 1934). Este material se encuentra depositado en el Museo de Historia Natural de los Estados Unidos (NMNH, 2011).

En julio de 1978, V. P. Vicente realizó colectas de esponjas en varias localidades de la costa Sur Dominicana (Boca Chica, La Malena y Las Salinas) hasta unos $6 \mathrm{~m}$ de profundidad, incorporando al menos siete nuevos registros (Vicente y Bonnelly, 1979), que también están depositados en el Museo de Historia Natural de los Estados Unidos (NMNH, 2011). En la siguiente década se ubica el trabajo de Rathe (1981) que lista unas 25 especies para República Dominicana, incluyendo nueve nuevos reportes; y la Expedición del B/I Crawford, que realizó inventarios en los arrecifes del Sureste dominicano en La Caleta y las Islas Catalina y Saona (Williams et al., 1983), aportando cinco nuevos registros.

Pero la contribución más importante al conocimiento de los poríferos dominicanos de la década de los 80 corresponde al trabajo de Pulitzer-Finali (1986) que resume sus muestreos realizados en abril de 1964 en cinco localidades (Boca Chica, La Caleta, Punta Magdalena, Punta Salinas y Sosúa). Este trabajo arrojó 53 nuevos reportes, incluida la descripción de cuatro especies reconocidas que tienen su localidad tipo en República Dominicana: Polymastia tenax (probablemente una sinonimia posterior de P. nigra en Alcolado, 1984) en Sosúa, y Xestospongia dominicana, Prosuberites psammophilus y Ecionemia dominicana (sinonimia posterior de Ancorina megastylifera en Wintermann-Kilian y Kilian, 1984) en Boca Chica. Asimismo, en junio de 1988 se realizó un detallado estudio de los arrecifes coralinos de Les Arcadines en Haití, en trece estaciones sobre diferentes zonas ecológicas (desde la laguna arrecifal hasta el arrecife frontal) en profundidades de 0.3 a $21 \mathrm{~m}$ (Wilcox et al., 1989), que reportó el mayor número de especies conocidas para aguas haitianas, con 58 especies de esponjas someras comunes.

Nuevos estudios, en la década de 1990, contribuyeron a ampliar el inventario de las esponjas dominicanas en diferentes ambientes arrecifales con nueve nuevos registros. Aquí se incluyen las investigaciones de la Universidad de Carolina del Este (Luczkovich, 1991) y CIBIMA (1998) entre Manzanillo y Punta Rucia en Montecristi, y del Caribbean Marine Conservation Science Center en el Parque Nacional del Este (CMCS, 1994). El trabajo del Centro para la Conservación y Ecodesarrollo de la Bahía de Samaná y su Entorno (CEBSE) ofrece el primer inventario de las esponjas de los arrecifes al Norte de la Península de Samaná (Sang, 1996).

Posteriormente tuvieron lugar importantes aportes al conocimiento de los poríferos dominicanos derivados de las investigaciones de The Nature Conservancy en el Parque Nacional del Este (TNC, 2001) y la Universidad de Puerto Rico en varias localidades (Grumelandia, Playa del Coco, Los Carraplanes, Punta Lanza, Cabo Falso, Lanza Zo, Bahía de Águilas, Bahía Honda en Cabo Rojo y Los Frailes) del Parque Nacional Jaragua, en Pedernales (Weil, 2006), que arrojaron 13 y 23 nuevos reportes de esponjas, respectivamente, para aguas dominicanas. A ello se suman tres nuevos reportes provenientes de los estudios del Programa EcoMar en Haina (Herrera-Moreno et al., 2009) y de la Universidad de la Florida en Pedernales (León y Bjorndal, 2002). La Universidad de Miami ofrece un inventario de las esponjas de los arrecifes de Bávaro y Punta Cana (Brandt et al., 2003). Grace et al. (2000), realizaron un estudio preliminar de la biota de Isla Navassa, donde listan tres especies de esponjas para esta localidad haitiana.

Se mencionan especies para República Dominicana en las revisiones del Orden Halichondrida (Díaz et al., 1993), las Familias Chalinidae (Weerdt et al., 1991; Weerdt, 2000), Axinellidae 
(Álvarez et al., 1998) y Tethyidae (Sarà, 2002), y los Géneros Mycale (Hajdu y Rützler 1998), Lissodendoryx (Rützler et al., 2007) e Iotrochota (Rützler et al., 2007a). Reiswig (2002), en su revisión de la Familia Aulocystidae, y Uriz (2002), en su revisión de la Familia Ancorinidae, mencionan sendas especies para Haití.

Al menos unas 41 especies de poríferos de Hispaniola están conservadas en las colecciones de cinco museos: 23 en el Museo de Historia Natural de los Estados Unidos (NMNH, 2011), ocho en el Museo de Génova MSNG (Pulitzer-Finali, 1986), siete en el Museo de Zoología Comparativa de Michigan (MCZ, 2011), dos en el Museo Zoológico de Ámsterdam (ZMA, 2011) y una en el Museo de Historia Natural de Gran Bretaña BMNH (Reiswig, 2002).

El número de esponjas conocidas para Hispaniola alcanza en esta revisión 138 especies (con dos formas), distribuidas en tres clases, 15 órdenes, 43 familias y 78 géneros. Se reportan 63 especies para Haití, 127 para República Dominicana (con 53 especies presentes en las aguas de ambos países) y una reportada para la Isla Hispaniola, sin aclarar en qué país fue colectada (Tabla 1). La presente compilación añade 102 especies a la revisión dominicana de CIBIMA

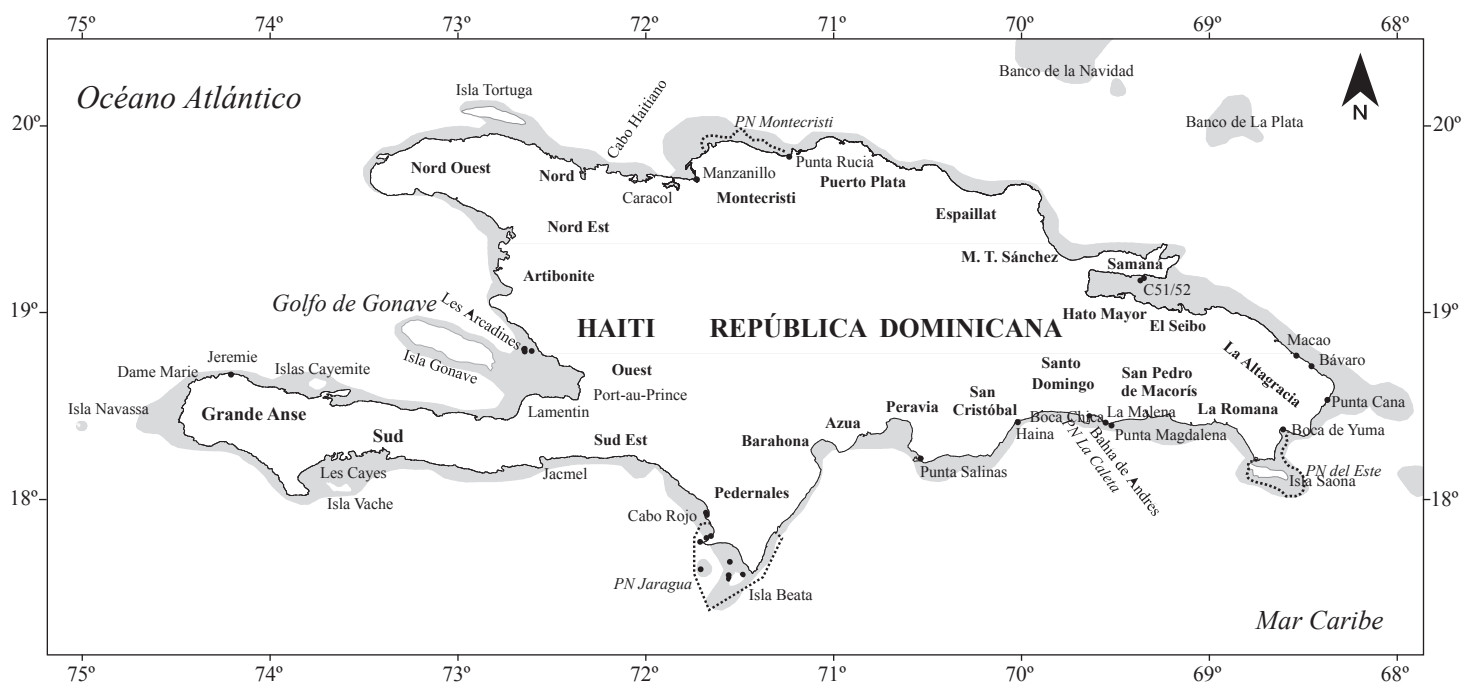

Figura 1. Mapa de la Isla Hispaniola mostrando las localidades de colecta de poríferos. La línea punteada indica el límite de los Parques Nacionales (PN). C51/52: Estaciones del Caroline en la Bahía de Samaná.

(1992), incluyendo seis especies con localidades tipo dominicanas. Todas las localidades que se mencionan en el texto se encuentran en el mapa de la Figura 1.

Tabla 1. Lista de poríferos de Hispaniola. *Especie tipo. D, República Dominicana. H, Haití. HI, Hispaniola sin aclaración de país. Se indican entre corchetes las referencias y con notas al pie las actualizaciones taxonómicas o comentarios.

Clase HEXACTINELLIDA

Orden Lychniscosida

Familia Aulocystidae

Neoaulocystis grayi (Bowerbank, 1869) H[Soest y Stentoft, 1988; BMNH 1910.10.18.3']

1 Como Aulocystis grayi. Ver revisión de Aulocystidae de Reiswig (2002). 


\section{Clase DEMOSPONGIAE}

Orden Homoscleromorpha

Familia Plakinidae

Plakortis angulospiculatus (Carter, 1879) D[Pulitzer-Finali, 1986²]/H[Wilcox et al., 1989]

Plakinastrella onkodes Uliczka, 1929 D[Pulitzer-Finali, 1986³]

Plakortis halichondrioides (Wilson, 1902) D[Weil, 2006]

Orden Spirophorida

Familia Tetillidae

Cinachyrella alloclada (Uliczka, 1929) D[Rathe, 1981]/H[Wilcox et al., 19894]

Cinachyrella kuekenthali (Uliczka, 1929) D[León y Bjorndal, 2002; NMNH3225655]

Orden Astrophorida

Familia Ancorinidae

Asteropus simplex (Carter, 1879) H[Carter, 18796]

Ecionemia dominicana (Pulitzer-Finali, 1986)* D[Pulitzer-Finali, 1986; Holotipo MSNG4767977]

Stelletta kallitetilla (de Laubenfels, 1936) D[León y Bjorndal, 2002]

Familia Calthropellidae

Pachastrissa hartmeyeri Uliczka, 1929 D[Pulitzer-Finali, 1986]

Familia Geodiidae

Erylus bahamensis Pulitzer-Finali, 1986 D[Pulitzer-Finali, 1986]

Erylus formosus Sollas, 1886 D[Williams et al., 1983]/ H[Wilcox et al., 1989]

Geodia gibberosa Lamarck, 1815 D[NMNH, 2011; NMNH22349 ${ }^{8}$ ]

Geodia neptuni (Sollas, 1888) D[Pulitzer-Finali, 1986]/H[Wilcox et al., 1989]

Orden Hadromerida

Familia Suberitidae

Prosuberites psammophilus (Pulitzer-Finali, 1986)* D[Pulitzer-Finali, 1986; Holotipo MSNG47686

Familia Polymastiidae

Polymastia tenax Pulitzer-Finali, 1986* D[Pulitzer-Finali, 1986; Holotipo MSNG47687]

Familia Chondrillidae

Chondrilla caribensis Rutzler, Durán y Piantoni, 2007 D[Vicente y Bonnelly, 1979]/H[Wilcox et al., 1989 $]^{10}$

2 Como Plakortis simplex

3 Como Dercitopsis onkodes

4 Como Cinachyra alloclada

5 Como Cinachyra kuekenthali

6 Como Stellettinopsis simplex

7 Como Stellettinopsis dominicana

8 En los datos del NMNH esta especie se ubica en Haití, pero las Coordenadas que se indican (19.16806 y -69.35694) corresponden a la Estación 52 de la Expedición Johnson-Smithsonian Deep Sea de 1933 en la Bahía de Samaná en República Dominicana.

9 Como Laxosuberites psammophilus

10 Como Chondrilla nucula en todas las fuentes. C. nucula fue descrita por primera vez por Schmidt (1862) para el Mediterráneo y se sospechaba que la población del Atlántico Occidental podía ser una especie separada, lo cual demuestran Rützler et al. (2007b) a partir de evidencia morfológica y molecular describiéndola bajo el nuevo nombre de Chondrilla caribensis, con dos formas ecológicas 
Familia Clionaidae

Cliona aprica Pang, 1973 D[Weil, 2006]/H[Wilcox et al., 1989'11]

Cliona delitrix Pang, 1973 D[Luczkovich, 1991]/H[Wilcox et al., 1989]

Cliona caribbaea Carter, 1882 D[TNC, 20012]

Cliona varians (Duchassaing y Michelotti, 1864) D[Pulitzer-Finali, 1986 ${ }^{13}$ ]

Cervicornia cuspidifera (Lamarck, 1815) D[Pulitzer-Finali, 1986 $\left.{ }^{14}\right] / \mathrm{H}[$ Wilcox et al., 1989]

Pione lampa (Laubenfels, 1950) D[Weil, 2006]

Spheciospongia vesparium (Lamarck, 1815) D[Pulitzer-Finali, 1986]

Familia Spirastrellidae

Spirastrella coccinea (Duchassaing y Michelotti, 1864) D[Pulitzer-Finali, 1986]

Familia Trachycladidae

Trachycladus spinispirulifer (Carter, 1879) D[Pulitzer-Finali, 1986 $\left.{ }^{15}\right]$

Familia Tethyidae

Tectitethya crypta (Laubenfels, 1949) D[Pulitzer-Finali, 1986]/H[Wilcox et al., 1989 ${ }^{16}$ ]

Tethya actinia Laubenfels, 1950 D[Vicente y Bonnelly, 1979]

Tethya aurantium (Pallas, 1766) D[Pulitzer-Finali, 1986]

Tethya diploderma Schmidt, 1870 D[TNC, 2001]

Familia Timeidae

Diplastrella megastellata Hechtel, 1965 D[TNC, 2001]

Timea unistellata (Topsent, 1892) D[Pulitzer-Finali, 1986]

Orden Agelasida

Familia Agelasidae

Agelas clathrodes (Schmidt, 1870) D[Pulitzer-Finali, 1986]/H[Wilcox et al., 1989]

Agelas conifera (Schmidt, 1870) D[Pulitzer-Finali, 1986]/H[Wilcox et al., 1989]

Agelas dispar Duchassaing y Michelotti, 1864 D[Pulitzer-Finali, 1986]/H[Wilcox et al., 1989]

Agelas sceptrum (Lamarck, 1815) D[TNC, 2001]/H[Wilcox et al., 1989]

Agelas schmidtii Wilson, 1902 D[Williams et al., 1983]/H[Wilcox et al., 1989]

Agelas weidenmayeri Alcolado, 1984 D[TNC, 2001]

Orden Poecilosclerida

Familia Acarnidae

Acarnus innominatus Gray, 1867 D[Pulitzer-Finali, 1986]

Familia Microcionidae

11 Como Cliona aprica forma profunda

12 Como Cliona langae

13 Como Anthosigmella varians

14 Como Spheciospongia cuspidifera

15 Como Spirastrella spinispirulifera

16 Como Tethya crypta en todas las referencias. En el material examinado por Sarà (2002) para la descripción de esta especie aparece: San Domingo. Pansini coll. 2206 
Artemisina melana Soest, 1984 D[Weil, 2006]

Clathria virgultosa (Lamarck, 1814) D[Pulitzer-Finali, 1986 ${ }^{17}$; NMNH32262 ${ }^{18}$ ] H[Wilcox et al., 1989]

Clathria (Microciona) bulbotoxa Soest, 1984 D[Pulitzer-Finali, 1986 ${ }^{19}$ ]

Pandaros acanthifolium Duchassaing y Michelotti, 1864 D[Luczkovich, 1991]/H[Wilcox et al., 1989]

Familia Raspailiidae

Ectyoplasia ferox (Duchassaing y Michelotti, 1864) D[Pulitzer-Finali, 1986; NMNH32259]/ H[Wilcox et al., 1989]

Familia Anchinoidae

Phorbas amaranthus Duchassaing y Michelotti, 1864 D[Weil, 2006]

Familia Crambidae

Monanchora arbuscula (Duchassaing y Michelotti, 1864) D[TNC, 200120]

Familia Myxillidae

Pseudotrachya amaza (de Laubenfels, 1934)* D[De Laubenfels, 1934; NMNH22348²1]

Desmapsamma anchorata Carter, 1882 D[Pulitzer-Finali, 198622; NMNH32257]/H[Wilcox et al., 1989]

Iotrochota birotulata (Higgin, 1877) D[Pulitzer-Finali, 1986]/ H[Wilcox et al., 1989]

Lissodendoryx (Lissodendoryx) isodictyalis (Carter, 1882) D[Rutzler et al., 2007a; NMNH32254]

Strongylacidon sp. D[Weil, 2006]

Familia Phloeodictyidae

Oceanapia fistulosa (Bowerbank, 1873) D[Pulitzer-Finali, 1986]

Familia Tedaniidae

Tedania ignis (Duchassaing y Michelotti, 1864) D[Pulitzer-Finali, 1986]

Familia Desmacellidae

Desmacella meliorata Wiedenmayer, 1977 D[Vicente y Bonnelly, 1979; NMNH32249]

Neofibularia nolitangere (Duchassaing y Michelotti, 1864) D[Pulitzer-Finali, 1986]/ H[Wilcox et al., 1989]

Familia Mycalidae

Mycale arndti Soest, 1984 D[Weil, 2006]

Mycale (Mycale) laevis Carter, 1882 D[Pulitzer-Finali, 1986²3]/H[Wilcox et al., 1989]

Mycale laxissima (Duchassaing y Michelotti, 1864) D[Weil, 2006; NMNH3225024]/ H[Wilcox et al., 1989]

Mycale (Grapelia) unguifera Hajdu, Zea, Kielman y Peixinho, 1995 D[TNC, 200125]/H[Wilcox et al., 1989]

Orden Halichondrida

Familia Axinellidae

17 Como Rhaphidophlus juniperinus

18 Como Thalysias juniperina

19 Como Microciona bulbotoxa

20 TNC (2001) incluye a Monanchora unguifera y M. barbadensis

21 Como Anomolissa amaza

22 Como Holopsamma helwigi. En el NMNH existe un ejemplar de Desmacidum carterianum (NMNH 22344) colectado en la

Bahía de Samaná. Según Hechtel (1965) esta especie es sinónima posterior de Desmapsamma anchorata

23 Como Oxymycale strongylata

24 Como Mycale (Acamisina) cf. laxissima

25 Como Mycale unguifera 
Axinella corrugata (George y Wilson, 1919)* D[De Laubenfels, 1934; NMNH2234726]

Dragmacidon reticulatum (Ridley y Dendy, 1886) D[P. Alcolado en Herrera-Moreno et al., 2009]/ H[Wilcox et al., 1989]

Dragmacidon lunaecharta (Ridley y Dendy, 1886) D[Pulitzer-Finali, 1986 ${ }^{27}$ ]

Ptilocaulis spiculifer (Lamarck, 1814) D[Pulitzer-Finali, 1986]/H[Wilcox et al., 1989]

Ptilocaulis walpersi (Duchassaing y Michelotti, 1864) D[NMNH, 2011; NMNH32266]

Familia Desmoxyidae

Myrmekioderma rea (de Laubenfels, 1934) D[Pulitzer-Finali, 1986; NMNH32265²8]

Familia Dictyonellidae

Dyctionella funicularis (Ruezler, 1981) D[Weil, 2006]/H[Wilcox et al., 198929]

Scopalina hispida (Hechtel, 1965) D[Williams et al., 19830]

Scopalina ruetzleri (Wiedenmayer, 1977) D[Rathe, 1981]

Svenzea zeai (Álvarez, Soest y Rützler, 1998) D[P. Alcolado en Herrera-Moreno et al., 2009]

Familia Halichondriidae

Axinyssa ambrosia (de Laubenfels, 1954) D[Pulitzer-Finali, 1986; MSNG47694¹3

Ciocalypta alleni de Laubenfels, 1936 HI[Duchassaing y Michelotti, 186422]

Didiscus sp. D[TNC, 2001]

Halichondria melanadocia Laubenfels, 1936 D[CMCS, 1994]

Hymeniacidon caerulea Pulitzer-Finali, 1986 D[Pulitzer-Finali, 1986]

Hymeniacidon heliophila (Parker, 1910) D[Pulitzer-Finali, 1986]

Topsentia ophiraphidites (de Laubenfels, 1934) D[Pulitzer-Finali, 1986; MSNG 4769133]

Orden Haplosclerida

Familia Callyspongiidae

Callyspongia (Cladochalina) armigera (Duchassaing y Michelotti, 1864) D[Weil, 2006]

Callyspongia (Callyspongia) eschrichti Duchassaing y Michelotti, 1864 H[Wilcox et al., 198934]

Callyspongia (Callyspongia) fallax (Duchassaing y Michelotti, 1864) D[Vicente y Bonnelly, 197935; NMNH32261]/H[Wilcox et al., 1989; ZMA4456; MCZ6468 ${ }^{36}$ ]

Callyspongia (Callyspongia) pallida Hetchel, 1965 D[CMCS, 1994 ${ }^{37}$ ]

Callyspongia (Cladochalina) plicifera (Lamarck, 1814) D[Rathe, 1981]/H[Wilcox et al., 198938]

Callyspongia (Callyspongia) simplex Burton, 1956 D[Pulitzer-Finali, 1986 ${ }^{39}$ ]

26 Como Oxeostilon burtoni

27 Como Pseudaxinella lunaecharta

28 Como Myrmekioderma styx en ambas referencias

29 Como Ulosa funicularis en Weil (2006) y Scopalina funicularis en Wilcox et al. (1989)

30 Como Dyctionella hispida

31 Como Dictyonella yumae

32 Como Spongia cavernosa. Dado que el reporte aparece para la Isla de Santo Domingo no queda claro a cual país corresponde.

33 Como Spongosorites sinuatus. Este material fue incluido en la revisión de Halichondrida de Díaz et al. (1993)

34 Como Callyspongia eschrichti

35 Esta especie aparece como Toxochalina multiformis en Pulitzer-Finali (1986)

36 Como Siphonochalina bullata

37 Como Callyspongia pallida

38 Como Callyspongia plicifera en todas las referencias

39 Como Callyspongia simplex 
Callyspongia (Cladochalina) tenerrima (Duchassaing y Michelotti, 1864) D[Rathe, 198100]

Callyspongia (Cladochalina) vaginalis (Lamarck, 1814) D[Pulitzer-Finali, 1986 $\left.{ }^{41}\right] /$ H[Wilcox et al., 1989; MCZ4517 $7^{42}$ ]

Familia Chalinidae ${ }^{43}$

Chalinula molitba (de Laubenfels, 1949) H[Wilcox et al., 198944]

Haliclona (Reniera) implexiformis (Hechtel,1965) D[Weerdt et al., 199155]

Haliclona (Reniera) tubifera (George y Wilson, 1919) D[Williams et al., 1983 NMNH3225146]/ H[Wilcox et al., 1989]

Familia Niphatidae

Aka coralliphaga (Ruetzler, 1971) D[Luczkovich, 1991]

Aka siphona (Laubenfels, 1949) D[Williams et al., 1983]

Amphimedon caribica (Pulitzer-Finali, 1986) D[Pulitzer-Finali, 1986 $\left.6^{47}\right]$

Amphimedon compressa Duchassaing y Michelotti, 1864 ${ }^{48}$ H[Wilcox et al., 1989]

Amphimedon erina (Laubenfels, 1936) D[Weil, 2006]

Amphimedon viridis Duchassaing y Michelotti, 1864 D[Rathe, 1981]/H[Wilcox et al., 1989]

Cribrochalina infundibula Schmidt, 1870 D[Pulitzer-Finali, 1986]/H[Wilcox et al., 198949]

Niphates alba Soest, 1980 D[Pulitzer-Finali, 198650]

Niphates amorpha Wiedenmayer, 1977 D[Pulitzer-Finali, 1986; MSNG 47701851]

Niphates digitalis Lamarck, 1814 D[Pulitzer-Finali, 1986; NMNH32263]/H[Wilcox et al., 1989]

Niphates erecta Duchassaing y Michelotti, 1864 D[Pulitzer-Finali, 1986]/H[Wilcox et al., 1989]

Familia Phloeodictyidae

Calyx podatypa (de Laubenfels, 1934) D[Weil, 2006]

Oceanapia bartschi (Laubenfels, 1934) D[CIBIMA, 1998]

Oceanapia nodosa (George y Wilson, 1919) H[Wilcox et al., 198952]

Familia Petrosiidae

Petrosia (Petrosia) weinbergi Soest, 1980 D[Pulitzer-Finali, 1986]

Petrosia pellasarca (Laubenfels, 1934) D[Pulitzer-Finali, 1986]/H[Wilcox et al., 1989]

Xestospongia muta (Schmidt, 1870) D[Pulitzer-Finali, 1986]/H[Wilcox et al., 1989]

Xestospongia portoricensis Soest, 1980 D[TNC, 2001]

40 Como Callyspongia tenerrima

41 Como Callyspongia vaginalis

42 Como Siphonochalina papyracea

43 El Museo de Historia Natural de los Estados Unidos alberga con el número 22351 un ejemplar identificado como Haliclona monticulosa (Verrill, 1907) pero que no hemos incluido en esta lista. Esta especie es de identidad dudosa y no es reconocida en la literatura actual. El material de la colección puede tratarse de otra especie de Haliclona o de otro género de Haplosclerida y requiere revisión.

44 Como Haliclona molitba

45 Como Haliclona implexiformis

46 Como Haliclona hogarthi

47 Como Cribochalina caribica

48 Como Spongia rubens. Dado que el reporte aparece para la Isla de Santo Domingo no queda claro a cual país corresponde si bien

Wilcox et al. (1989) la reportan para Haití.

49 Como Cribochalina vasculum

50 Como Protophlitaspongia antillana

51 Pulitzer-Finali (1986) reporta a Niphates amorpha y su posible sinónima Gelliodes sosuae

52 Como Pellina nodosa 
Xestospongia proxima (Duchassaing y Michelotti, 1864) D[Weil, 2006]

Xestospongia wiedenmayeri Soest, 1980 D[CMCS, 1994]

Xestospongia carbonaria (Lamarck, 1813) D[Weil, 2006; NMNH32260]/ H[Wilcox et al., 198953]

Xestospongia dominicana (Pulitzer-Finali, 1986)* D[Pulitzer-Finali, 1986; MSNG47703]

Xestospongia subtriangularis (Duchassaing, 1850) D[Pulitzer-Finali, 1986] ${ }^{54} / \mathrm{H}\left[\mathrm{MCZ6445} 5^{55}\right]$

Orden Dictyoceratida

Familia Irciniidae

Hyrtios proteus Duchassaing y Michelotti, 1864 D[Weil, 2006]/H[Wilcox et al., 1989]

Hyrtios violaceus (Duchassaing y Michelotti, 1864) D[Luczkovich, 199156]

Ircinia campana (Lamarck, 1813) D[TNC, 2001]/H[Wilcox et al., 1989]

Ircinia felix (Duchassaing y Michelotti, 1864) D[Rathe, 1981]/H[Wilcox et al., 1989]

Ircinia strobilina (Lamarck, 1816) D[Vicente y Bonnelly, 1979]/H[Wilcox et al., 1989]

Smenospongia aurea (Hyatt, 1875) D[Pulitzer-Finali, 1986; NMNH32264] /H[Hyatt, 1877 ${ }^{57}$ ]

Smenospongia conulosa Pulitzer-Finali, 1986 D[Pulitzer-Finali, 1986; Paratipo MSNG 47712]

Familia Spongiidae

Hyattella cavernosa (Pallas, 1766) D[Weil, 2006 58]/H[ZMA, 2011; ZMA15307]

Spongia (Spongia) tubulifera Lamarck, 1814 H[Wilcox et al., 198959]

Spongia obscura Hyatt, 1877 D[Weil, 2006]

Spongia pertusa Hyatt, 1877 D[Weil, 2006]

Spongia (Spongia) graminea Hyatt, 1877 H[MCZ, 2011; MCZ6525]

Hippospongia gossypina (Duchassaing y Michelotti, 1864) H[Hyatt, 1877; MCZ716560]

Orden Dendroceratida

Familia Dysideidae

Dysidea etheria Laubenfels, 1936 D[TNC, 2001]/H[Wilcox et al., 1989]

Dysidea janiae (Duchassaing y Michelotti, 1864) D[Weil, 2006]

Familia Darwinellidae

Darwinella rosacea Hechtel, 1965 H[Wilcox et al., 1989]

Aplysilla glacialis (Merejkowski, 1878) D[Weil, 2006]

Orden Halisarcida

Familia Halisarcidae

Halisarca sp. D[Weil, 2006]

Orden Verongida

Familia Aplysinidae

Aplysina archeri (Higgin, 1825) D[TNC, 2001]/H[Wilcox et al., 1989]

53 Como Pellina carbonaria

54 Como Xestospongia subtriangularis

55 Como Schmidtia aulopora

56 Como Oligoceras hemorrhages

57 Como Stelospongos cribriformis

58 Como Hyattella intestinalis

59 Como Spongia tubulifera

60 Como Spongia lapidescens var. typica 
Aplysina cauliformis Carter, 1882 D[Rathe, 1981]/H[Wilcox et al., 1989]

Aplysina fistularis (Pallas, 1766) D[Vicente y Bonnelly, 1979]/H[Carter, 187961; MCZ61 ${ }^{62}$ ]

Aplysina fistularis forma insularis Duchassaing y Michelotti, 1864 H[Wilcox et al., 1989]

Aplysina fistularis forma fulva Pallas, 1766 D[Rathe, 1981]/H[Wilcox et al., 1989]

Aplysina lacunosa (Lamarck, 1814) D[Vicente y Bonnelly, 1979]/H[Wilcox et al., 1989]

Verongula gigantea (Hyatt, 1875) D[Pulitzer-Finali, 1986]/H[Wilcox et al., 1989]

Verongula rigida (Esper, 1794) D[TNC, 2001]/H[Wilcox et al., 1989]

Familia Druinellidae

Aiolochroia crassa (Hyatt, 1875) D[Pulitzer-Finali, 198663; NMNH2235264]/H[Wilcox et al., 1989]

Clase CALCAREA

Orden Clathrinida

Familia Clathrinidae

Clathrina coriacea (Montagu, 1936) D[CMCS, 1994]/H[Wilcox et al., 1989]

Orden Leucosolenida

Familia Grantiidae

Leucandra aspera (Schmidt, 1862) D[Weil, 2006]

Leucandra barbata (Duchassaing y Michelotti, 1864) D[Weil, 2006; NMNH2234565]

61 Como Luffaria fistularis

62 Como Verongia fistularis

63 Como Pseudoceratina crassa

64 En los datos del NMNH esta especie aparece como Ianthella ardis y se ubica en Haití, pero las Coordenadas que se indican

(19.16806 y -69.35694) corresponden a la Estación 52 de la Expedición Johnson-Smithsonian Deep Sea de 1933 en la Bahía de

Samaná en República Dominicana

65 Como Leuconia barbata

\section{LITERATURA CITADA}

Alcolado, P. M. 1984. Nuevas especies de esponjas encontradas en Cuba. Poeyana, 271: 1-22.

Alcolado, P. M. 2002. Catálogo de las esponjas de Cuba. Avicennia, 15: 53-72.

Álvarez, B., R. W. M. Van Soest y K. Rützler 1998. A Revision of Axinellidae (Porifera: Demospongiae) in the Central West Atlantic Region. Smithsonian Contributions to Zoology, 598: 1-47.

Brandt, M. E., W. T. Cooper y J. F. Polsenberg 2003. Results of a coral reef survey of Punta Cana, Dominican Republic, with comparisons to past studies and other Caribbean reefs, Agosto 20-25. Reporte de The National Center for Caribbean Coral Reef Research Rosenstiel School of Marine and Atmospheric Science University of Miami, 39 pp.

Caribbean Marine Conservation Science Center (CMCS).1994. Rapid ecological assessment, Parque Nacional del Este, Dominican Republic. Reporte del Caribbean Marine Conservation Science Center at the University of Miami, $395 \mathrm{pp}$.

Carter, H. J. 1879. Contributions to our knowledge of the Spongida. Annals and Magazine of Natural History (5) 3: 284-304, 343-360, pls. XXV-XXVII. 
Centro de Investigaciones de Biología Marina (CIBIMA). 1992. Estudio preliminar sobre la biodiversidad costera y marina de la República Dominicana. Editora Alfa y Omega, Centro de Investigaciones de Biología Marina, Universidad Autónoma de Santo Domingo, 459 pp.

Centro de Investigaciones de Biología Marina (CIBIMA). 1998. La diversidad biológica de los ecosistemas marinos del Parque Nacional de Montecristi, Reporte técnico final al proyecto GEF-PNUD/ONAPLAN: Conservación y Manejo de Biodiversidad de la Zona Costera de la República Dominicana, Centro de Investigaciones de Biología Marina, Universidad Autónoma de Santo Domingo, 36 pp.

Díaz, M. C., S. A. Pomponi, y R. W. M. Van Soest. 1993. A systematic revision of the central West Atlantic Halichondrida (Demospongiae, Porifera). Part III: Description of valid species. Pp. 283-306. En: Uriz, M.-J. y K. Rützler (Editores), Recent Advances in Ecology and Systematics of Sponges. Scientia Marina, 57(4): 273-274.

Duchassaing De Fonbressin, P y G. Michelotti. 1864. Espongiaires de la mer Caraïbe. Natuurkundige verhandelingen van de Hollandsche maatschappij der wetenschappen te Haarlem 21(2): 1-124, pls. I-XXV.

Grace, M., M. Bahnick y L. Jones. 2000. A preliminary study of the marine biota at Navassa Island, Caribbean Sea. Marine Fisheries Review, 62(2): 43-48.

Hajdu E. y K. Rützler. 1998. Sponges, genus Mycale (Poecilosclerida: Demospongiae: Porifera), from a Caribbean mangrove and comments on subgeneric classification Proceedings of the Biological Society Of Washington, 111(4):737-773.

Hechtel, J. G. 1965. A systematic study of the Demospongiae of Port Royal, Jamaica. Peabody Museaum of Natural History, 20: 103 pp.

Herrera-Moreno, A. y L. Betancourt. 2005. Inventario de la biota marina de la Hispaniola. Ciencia y Sociedad, 30 (1):158-167.

Herrera-Moreno, A. y L. Betancourt. 2011. Hispabiota Marina Project: the first inventory of the marine biota of Hispaniola Island. Programa EcoMar, Inc. Santo Domingo. Disponible en: http://programaecomar.com/HISPABIOTAMARINA.htm

Herrera-Moreno, A., L. Betancourt y P. M. Alcolado. 2009. Impacto de la contaminación sobre los arrecifes coralinos al Oeste del Río Haina, San Cristóbal, República Dominicana. Reporte del Proyecto Impactos a los arrecifes dominicanos, Programa EcoMar, Santo Domingo, 15 pp. Disponible en: http://programaecomar.com/Arrecifes_Haina.pdf

Hyatt, A. 1877. Revision of the North American Poriferae; with Remarks upon Foreign Species. Part II. Memoirs of the Boston Society of Natural History 2: 481-554, pls. XV-XVII.

Laubenfels, M. W. De. 1934. New sponges from the Puerto Rican deep. Smithson. Misc. Coll. 91 (17), 28 pp.

Laubenfels, M. W. De 1936. A Discussion of the Sponge Fauna of the Dry Tortugas in Particular and the West Indies in General, with Material for a Revision of the Families and Orders of the Porifera. Carnegie Institute of Washington (Tortugas Laboratory Paper $\mathrm{N}^{\circ} 467$ ) 30: $1-225$, pls. $1-22$. 
León, Y. y K. A. Bjorndal. 2002. Selective feeding in the hawksbill turtle, an important predator in coral reef ecosystems. Mar. Ecol. Prog. Ser., 245: 249-258.

Luczkovich, J. J. 1991. Marine Ecology of the Buen Hombre Coast. In: Satellite monitoring of coastal marine ecosystems: a case from the Dominican Republic, R. W. Stoffle y D. B. Halmo, eds., East Carolina University, pp. 93- 141.

Museum of Comparative Zoology (MCZ). 2011. Harvard University, Museum of Comparative Zoology MCZ, Marine Invertebrate Collections. Disponible en: http://collections.mcz. harvard.edu/MarineInvert/MarineInvertSearch.html (Accesado el 3 de abril de 2011).

National Museum of Natural History (NMNH). 2011. National Museum of Natural History, Smithsonian Institution. Department of Invertebrate Zoology. Disponible en: http:// collections.nmnh.si.edu/search/iz/ (Accesado el 3 de abril de 2011).

Pulitzer-Finali, G. 1986. A collection of West Indian Demospongiae (Porifera). In appendix, a list of the Demospongiae hitherto recorded from the West Indies. Annali del Museo civico di storia naturale Giacomo Doria 86: 65-216.

Rathe L. 1981. Estudio sistemático de las esponjas (Porifera) del litoral de la República Dominicana. Tesis de grado, Departamento de Biología, Universidad Autónoma de Santo Domingo, República Dominicana.

Reiswig H. M. 2002. Familia Aulocystidae Sollas, 1887. En: Systema Porifera: A Guide to the Classification of Sponges, Editado por: J. N. A. Hooper y R. W. M. Van Soest, Kluwer Academic/Plenum Publishers, New York, pp. 1378-1382.

Rützler K., C. Piantoni y M. C. Díaz. 2007. Lissodendoryx: rediscovered type and new tropical western Atlantic species (Porifera: Demospongiae: Poecilosclerida: Coelosphaeridae). J. Mar. Biol. Ass. U.K., 87, 1491-1510.

Rützler K., M. Maldonado, C. Piantoni y A. Riesgo. 2007a. Iotrochota revisited: a new sponge and review of species from the western tropical Atlantic (Poecilosclerida: Iotrochotidae). Invertebrate Systematics, 21, 173-185.

Rützler, K., S. Duran y C. Piantoni. 2007b. Adaptation of reef and mangrove sponges to stress: evidence for ecological speciation exemplified by Chondrilla caribensis new species (Demospongiae, Chondrosida). Marine Ecology 28 (Suppl.1): 95-111.

Sang, L. 1996. Estudio de los arrecifes de coral de la costa Norte de la Península de Samaná. Proyecto inventario de la biodiversidad y caracterización de las comunidades del entorno de la Península y Bahía de Samaná, Centro para la conservación y Ecodesarrollo de la Bahía de Samaná y su Entorno, CEBSE, Inc. 97 pp.

Sarà M. 2002. Family Tethyidae Gray, 1848. En: Systema Porifera: A Guide to the Classification of Sponges, Editado por: J. N. A. Hooper y R. W. M. Van Soest, Kluwer Academic/Plenum Publishers, New York, pp. 245-265.

Schmidt, O. 1862. Die Spongien des adriatischen Meeres. (Wilhelm Engelmann: Leipzig): i-viii, 1-88, pls. $1-7$. 
Soest, R. W. M. van, N. Boury-Esnault, J. N. A. Hooper, K. Rützler, N. J de Voogd, B. Álvarez, E. Hajdu, A. B. Pisera, J. Vacelet, R. Manconi, C. Schoenberg, D. Janussen, K. R. Tabachnick y M. Klautau. 2011. World Porifera Database. Disponible en: http://www.marinespecies. org/porifera (Accesado el 23 de octubre de 2011).

Soest, R. W. M. van y N. Stentoft. 1988. Barbados deep-water sponges. Stud. Fauna Curacao Caribb. Isl. 70(215): 175 pp.

Soest, R. W. M. van, S. M. Stone, N. Boury-Esnault y K. Rützler. 1983. Catalogue of the Duchassaing y Michelotti (1864) collection of West Indian sponges (Porifera). Bull. Zool. Mus. Univ. Amsterdam 9 (21): 189-205.

The Nature Conservancy (TNC). 2001. Coral Reef Conservation in Marine Protected Areas: A Case Study of Parque Nacional del Este, Dominican Republic. Editado por Mark Chiappone, The Nature Conservancy, $244 \mathrm{pp}$.

Uriz M. J. 2002. Family Ancorinidae Schmidt, 1870. Systema Porifera: A Guide to the Classification of Sponges, Editado por: J. N. A. Hooper y R. W. M. Van Soest, Kluwer Academic/Plenum Publishers, New York, 19 pp.

Vicente, V. P. e I. Bonnelly 1979. Nuevo record de esponjas marinas para la República Dominicana y discusión de su importancia en el ambiente marino. Proc. Assoc. Is. Mar. Labs. Caribb., 14: 8.

Weerdt, W. H. 2000. A monograph of the shallow-water Chalinidae (Porifera, Haplosclerida) of the Caribbean. Beaufortia 50(1): 1-67.

Weerdt, W. H., K. Rutzler y K. P. Smith. 1991. The Chalinidae (Porifera) of Twin Cays, Belize, and adjacent waters. Proceedings of the Biological Society of Washington 104: 189-205.

Weil, E. 2006. Diversidad y abundancia relativa de corales, octocorales y esponjas en el Parque Nacional Jaragua, República Dominicana. Rev. Biol. Trop., 54 (2): 423-443.

Wilcox, E., T. Deyo, A. Gardella, R. García, D. Glick, C. Goneaga, A. Medina, V. Vicente y E. Wilcox. 1989. Proposed Les Arcadins National Marine Park resource document. World Wildlife Fund. Conservation Foundation Wilcox Associates, 102 pp.

Williams, E. H., I. Clavijo, J. J. Kimmel, P. L. Colin, C. Díaz, A. T. Bardales, R. A. Armstrong, L. Bunkley, R. H. Boulon y J. R. García. 1983. A checklist of marine plants and animals of the south coast of the Dominican Republic. Carib. J. Sci. 19 (1-2): 39-54.

Wintermann-Kilian, G. y E. F. Kilian. 1984. Marine Sponges of the Region of Santa Marta (Colombia). Part II. Homosclerophorida, Choristida, Spirophorida, Hadromerida, Axinellida, Halichondrida, Poecilosclerida. Studies on Neotropical Fauna and Environment, Alblasserdam 19 (3): 121-135.

Zoological Museum Amsterdam (ZMA). 2011. Porifera collection of ZMA. Disponible en: http://ip30.eti.uva.nl/zmawebsite/search_in_collection.php?collection=Porifera (Accesado el 3 de abril de 2011). 